\title{
Les objets sont notre plomb dans la tête. Efficacités en actions, innovations en usages
}

Philippe Geslin

\section{(2) OpenEdition \\ 12 Journals}

Édition électronique

URL : https://journals.openedition.org/tc/1444

DOI : $10.4000 /$ tc. 1444

ISSN : 1952-420X

Éditeur

Éditions de l'EHESS

\section{Édition imprimée}

Date de publication : 1 avril 2003

ISSN : 0248-6016

\section{Référence électronique}

Philippe Geslin, « Les objets sont notre plomb dans la tête. Efficacités en actions, innovations en usages », Techniques \& Culture [En ligne], 40 | 2003, mis en ligne le 18 mai 2006, consulté le 29

septembre 2022. URL : http://journals.openedition.org/tc/1444; DOI : https://doi.org/10.4000/tc.1444

Ce document a été généré automatiquement le 29 septembre 2022.

Tous droits réservés 


\title{
Les objets sont notre plomb dans la tête. Efficacités en actions, innovations en usages
}

\author{
Philippe Geslin
}

1 Renouant avec les périodes pas si lointaines des joutes intellectuelles par papiers interposés, Pierre Lemonnier et Bruno Latour se livrèrent en 1996 à un échange fructueux entre d'un côté une ethnologie des techniques aux origines maussiennes et de l'autre une sociologie des sciences et des techniques fortement influencée par le mouvement des "social studies of knowledge». Entre «Et pourtant ça vole! L'ethnologie des techniques et les objets industriels » et « Lettre à mon ami Pierre sur l'anthropologie symétrique ", nous aurions souhaité que l'ébauche se poursuive d'un renouvellement conceptuel attendu en technologie culturelle ou en ethnologie des techniques ${ }^{1}$. L'apport n'était pas symétrique, lui qui portait sur le traitement justement symétrique des humains et des non-humains. L'ouvrage dirigé deux ans plus tôt par ces auteurs, De la préhistoire aux missiles balistiques, marquait pourtant une étape importante dans le rapprochement de ces champs disciplinaires. Plus de clivage entre le "proche " et le « lointain ». Ethnologues et sociologues se retrouvaient ainsi autour des manières qu'ils avaient de saisir l'élaboration simultanée du lien social et des innombrables objets avec lesquels nous partageons notre existence (Latour \& Lemonnier 1994 : 10).

2 La sociologie des sciences et des techniques semblait apporter plus qu'elle ne recevait de l'ethnologie. Ouverte aux ethno-méthodes et à certains courants des sciences cognitives, la première nous faisait découvrir d'autres formes de traitement de ces objets qui nous entourent, un peu à la manière d'un Francis Ponge qui, prenant le parti des choses, leur accordait en son temps une place de choix dans le monde littéraire. Quel plus bel hommage -d'ailleurs toujours d'actualité pour les ethnologues- que celui qui lui est rendu par l'écrivain André Pieyre de Mandiargues :

«[...] la nouveauté grande que je vois dans [la poésie] de F. Ponge est de n'être point bornée à refléter uniquement le feu, mais de se faire le témoin et l'écho de la pierre (depuis le bloc originel jusqu'au caillou, à l'éclat, au gravier et au gravillon le plus menu).» (1956:102). 
3 Le rapport de l'homme à l'objet, nous dit Ponge (1962), n'est du tout seulement de possession ou d'usage; les objets sont notre plomb dans la tête. Les sociologues l'avaient sans doute oubliés ${ }^{2}$, pas les ethnologues à qui on ne la faisait plus et dont la constance de leur entreprise somme toute ancienne méritait le respect qu'on accorde aux aînés dont on a beaucoup à apprendre.

4 Les échanges auraient pu se situer sur la relation du social à la technique, mais les débats auraient très certainement tourné court, renvoyant d'un côté à la critique d'un dualisme révolu dont on peut douter qu'il ait jamais existé chez les ethnologues des techniques, et de l'autre à celle du «tissu sans couture ", image utilisée pour illustrer l'impossibilité qu'il y a à dissocier dans tout système technologique des pièces d'une texture uniforme, que celle-ci soit sociale, technique ou économique (Akrich 1994 : 123). Il ne nous appartient pas ici de trancher le débat, tout au plus pouvons-nous renvoyer aux analyses auxquelles cette controverse a donné lieu, dont celles des anthropologues Segalen et Bromberger (1996: 9) et des historiens Cohen et Pestre (1998 : 729). Les premiers parlent de malentendu, soulignant que les distinctions entre instances opérées par Lemonnier sont avant tout d'ordre méthodologique (isoler d'abord pour mieux conjoindre ensuite), et d'effet d'échelle. Les seconds précisent que si l'on postule que la dichotomie initiale a un sens - il y a la nature et ses lois d'une part, la société dans laquelle s'élaborent les savoirs, de l'autre- et qu'on cherche à juger du poids relatif des deux déterminations, alors Lemonnier doit avoir raison... Si l'on admet, en revanche, qu'une réponse plus précise à cette question est impossible la pondération relative $\mathrm{du}$ " naturel » et $\mathrm{du}$ " social » ne peut être évaluée- et que c'est donc plutôt aux formes concrètes de la mise en forme de l'objet par l'humain et le social qu'il convient de s'intéresser, alors Latour doit avoir raison. C'est sur le terrain de l'efficacité que la rencontre eut lieu. Lemonnier souligne :

«Le désaccord maximum [entre la technologie culturelle et la nouvelle sociologie des sciences et des techniques] concerne l'idée d'efficacité qui revient comme un leitmotiv dans les études de technologie culturelle mais que les sociologues de l'innovation choisissent d'ignorer. » (1996: 17).

Alors que B. Latour, en quelques mots, rejette de telles remarques :

«Quant à l'efficacité technique, elle me paraît tellement évidente que je ne comprends même pas comment on peut vouloir la souligner, sauf bien sûr, contre ceux qui se font une idée du monde social si évaporée, si remplie de signes, qu'il faut, en tapant du poing sur la table, les faire redescendre dans les choses. » (1996: 33).

6 Ce débat sur l'efficacité nous laissait sur notre faim. Dès 1996, nous avions engagé une ébauche de réflexion sur cette notion en nous inspirant des travaux d'ergonomes ${ }^{3}$. Nous proposions à l'époque d'aller plus avant dans ce que cette notion recouvre, sans, bien entendu, avoir la prétention d'épuiser le sujet, mais en dépassant l'évidence pour saisir un peu plus de son essence. C'est cette réflexion que je propose de poursuivre dans ce papier en partant d'un terrain de recherche/intervention ethnologique et ergonomique effectué dans un établissement de la Société Nationale des Chemins de Fer (SNCF). Ce terrain a la particularité de nous faire prendre conscience des limites que nous propose une approche de l'efficacité en termes de rapport de moyens à fin. Cette perception occidentale nous est familière. Elle projette un plan sur le cours des choses (Jullien $1996: 48)^{4}$. Les conduites résultent dans ce cas d'une application, quand en fait, dans notre exemple, elles sont plutôt à penser en termes d'exploitation ${ }^{5}$ c'est-àdire en tant qu'utilisation du potentiel impliqué dans la situation (1948: 50). Cette 
pensée de l'efficacité que nous rencontrons en Chine se rapproche en fait des travaux issus des sciences cognitives (i.e. Suchman 1987) qui mettent l'accent sur le rôle du contexte et de la situation dans la construction des connaissances au sein de collectifs d'acteurs, privilégiant ainsi l'action située au plan. Nous entrons dès lors dans une logique de déroulement. Le potentiel ne peut pas être déterminé d'avance. Il naît de la situation nous amenant aussi à reconsidérer le cours des choses, leur durée. Dans notre exemple, le TGV (Train à Grande Vitesse) venait de battre un record de vitesse. Même s'il ne s'agissait plus alors d'une innovation -ce train circulait depuis plusieurs années sur nos lignes nationales-, ce record réactivait l'image de cette prouesse technologique. Vivant de difficiles conditions de travail, le collectif d'acteurs impliqués dans la maintenance des amortisseurs a su tirer parti du potentiel de la situation d'alors, pour exprimer les maux auxquels il était confronté dans son activité quotidienne. À travers cet événement, le TGV redevient une innovation en usage -il est pour un temps plus rapide que les précédents- rompant avec les routines habituelles auxquelles renvoient de simples techniques en usage ${ }^{6}$. Il crée ainsi une situation exceptionnelle, dont le potentiel va jouer en la faveur de l'équipe de maintenance.

Pins, Pin-up et TGV ou les cadres de l'action

7 L'action se déroule à trois kilomètres de la gare d'une ville de l'est de la France. L'établissement construit à la fin du XIXe siècle par l'administration allemande a vécu les aléas des guerres successives jusqu'à redevenir propriété de la SNCF en 1945. Rien ne semble avoir changé ici depuis sa construction. Les wagons dont les silhouettes se dessinent dans la brume matinale encombrent la cour, attendant leur restauration prochaine sur les voies de service qui desservent les différents ateliers, couvrant ainsi 25 ha. De l'extérieur, tout semble figé. Les lignes de métal, parallèles, disparaissent dans des bâtiments aux portes coulissantes, sortes de boîtes noires, posées sur les voies, où les 700 cheminots s'activent. Elles réapparaissent à l'autre extrémité, poursuivant leur chemin vers de nouvelles destinations. Les actes de maintenance doivent être efficaces. Délavées, vieillies, embouties ou taguées, les silhouettes élancées oranges et bleues des TGV Sud-Est et Atlantiques y sont objets d'attentions particulières. Fleuron de la Société Nationale des Chemins de Fer, les rames en ressortent restaurées, réparées, comme neuves.

8 Peu de temps avant notre arrivée, ce train qui se voudrait avion venait donc de battre un record de vitesse. Dans chacune des boites noires, les affiches relatant la performance fleurissaient sur les murs. La mode était aussi aux "pins ", à ceux du record, petits bouts de métal à la résine brillante et colorée. Objets fétiches aux formes du TGV, ils étaient épinglés sur les poitrines. Nous arrivâmes bientôt dans une autre boîte noire, celle où nous devions intervenir et qui est appelée dans le jargon des cheminots « chantier amortisseurs ». Moins rutilante, elle n'offrait aucune affiche du record, seulement les calendriers du fabricant de la marque d'amortisseurs « Koni » sur lesquels des pin-up vantaient en des poses suggestives les mérites de ces objets dont motrices et voitures ne peuvent se passer. Pas un "pins" sur les vêtements des compagnons de l'atelier. Pas une voiture de TGV, rien qui nous rappelle l'objet technique et ses prouesses en ce lieu, juste seize postes de travail dont les éclairages individuels compensent la faible lumière dispensée par l'unique baie encrassée. Les amortisseurs sont là sur $1100 \mathrm{~m}^{2}$, en cours de démontage, pissant l'huile, "désossés ", la peinture craquelée. Les caisses "arrivées " jouxtent les caisses "départ " sur les zones de stockage. Alignées, les premières font grise mine avec leur cargaison 
essoufflée, quand les secondes font de ce gris le symbole de l'œuvre achevée, traduction d'une renaissance, acte ultime de cette phase de maintenance, avant son remontage.

Ces amortisseurs sont hydrauliques. Sur les rames de TGV, ils sont montés sur les voitures et la motrice, mais aussi entre celles-ci. On les nomme alors «inter-caisses » ou « caisse-caisse ». Ils sont de formes, de tailles, de poids et de fonctions différentes. Ils garantissent le confort, la stabilité et la sécurité. Sur les rames, ils sont discrets. Horizontaux ou verticaux, le voyageur les remarque à peine. Ils ont aussi une durée de vie limitée, au terme de laquelle ils sont déposés puis acheminés dans cet atelier où l'on procède à leur maintenance, où ils reprennent vie. Dans leur usage, aucun détournement n'est possible. Chacun est un "symbole " et chaque "symbole " correspond à une fonction précise. Ils ont des fonctions et des emplacements spécifiques.

L'atelier n'est pas isolé des ateliers voisins. Son activité est étroitement dépendante de celles des ateliers de lavage, de tournage, de tôlerie et de peinture, auxquels il faut ajouter le magasin pour l'obtention de pièces neuves comme les joints et autres pièces impossibles à réparer. Quarante-trois éléments sont ainsi vérifiés, restaurés ou échangés et le temps de réparation est tel que dans certains cas, son coût est proche de celui d'un amortisseur neuf.

11 Chaque jour, les palettes d'amortisseurs usagés sont réceptionnées en "gare d'arrivée ». Puis, manuellement, où à l'aide d'une potence, chaque pièce est prélevée pour être positionnée verticalement sur l'étau où a lieu son démontage, sa vérification et la préparation des pièces qui suivront alors un itinéraire précis de poste de travail en poste de travail, avec à chaque fois des séries d'opérations qui finalement permettront son remontage. Après quoi, l'amortisseur sera testé, puis réglé sur le banc d'essai avant l'ultime vérification et son acheminement vers la « gare départ ».

12 Trente-deux compagnons se partagent les lieux selon des horaires variables et une organisation spécifique à la discrétion des chefs d'équipes. Lorsque les tâches ne sont pas achevées pour un amortisseur, le successeur prend la relève à l'embauche en suivant les consignes de son prédécesseur. Il n'est pas rare qu'un petit bout de papier traîne à proximité de l'amortisseur éventré, porteur d'un court message : «Je n'ai pas eu le temps de faire le complément de la Gira ». Le repas de midi se prend en commun, à la cantine, et l'on est frappé de voir les compagnons de cet atelier se regrouper et s'isoler du reste des compagnons. Le temps libre qui sépare la fin du repas de l'heure d'embauche est mis à profit pour un tournoi d'échecs qui se déroule tout au long de l'année entre les membres de l'atelier, toutes équipes confondues.

13 Rien ne distingue en apparence cet atelier de tous les autres, si ce n'est sa fonction spécifique, la réparation des amortisseurs. Ils arrivent et repartent en temps voulu. En d'autres lieux, ils sont remontés et les rames de TGV peuvent ainsi repartir, restaurées. Cette prouesse technologique peut reprendre du service, sans que les passagers que nous sommes n'imaginent un instant la quantité d'opérations et les travaux des compagnons à qui nous devons ce confort. Les TGV entrent en gare, l'innovation entre en gare, l'objet technique entre en gare, l'œuvre des concepteurs entre en gare, rarement celle des équipes de maintenance garantes de sa pérennité. Orange, bleu ou bordeaux, à chaque couleur son espace géographique, sa diversité de lieux, pour chaque amortisseur un gris unique, une présence discrète, d'une efficacité technique indéniable, mais socialement source de malaises. L'atelier de réparation des amortisseurs bénéficie en effet d'une mauvaise réputation. On évoque volontiers à son 
propos un contexte peu favorable. Y être affecté était vécu par les compagnons comme une punition, ne contribuant pas ainsi à le faire évoluer, mais au contraire à le considérer comme le parent pauvre de l'établissement. Les opérateurs de cet atelier souffrent d'une absence de reconnaissance de leur travail par leur hiérarchie, ce qui se traduit entre autres par un absentéisme important tout au long de l'année et par des formes de comportements sociaux à la marge de ceux qui ont cours au sein de l'établissement. Nous entrons là au cœur de la notion d'efficacité, sur ce qui, de l'esprit ou des propriétés intrinsèques de la matière, détermine fondamentalement les savoirs humains (Cohen et Pestre 1998 : 728).

Rendre compte des efficacités en actions dans les « innovations en usages »

À travers l'exemple de la maintenance, nous ne considérons pas le processus et les réseaux d'acteurs qui ont conduit au développement du TGV. Nous changeons d'échelle et accordons dans un premier temps une place prépondérante à "l'innovation en usage » (Edgerton 1998), à ses formes d'appropriation par ceux qui sont chargés de la faire vivre dans la société. Les comportements routiniers qu'elles impliquent (la réparation quotidienne des amortisseurs) sont autant de sources d'informations qui, nous allons le voir, permettent d'avancer sur le terrain de l'efficacité.

Cette notion d'innovation en usage est à différencier de celle de techniques en usage. Il existe en effet une période au cours de laquelle l'innovation n'est pas encore tout à fait stabilisée dans la société. C'était le cas notamment au moment de notre enquête. Le $\mathrm{TGV}$, nous l'avons dit, avait battu un nouveau record de vitesse. Il ne constituait plus tout à fait une innovation dans la mesure où il était en usage depuis plusieurs années, mais ses performances récentes traduisaient toutefois un progrès par rapport à un stade antérieur. Il n'était pas encore une simple technique en usage avec les comportements de routines qu'elle entraîne.

Les phases au cours desquelles il est encore possible de parler d'innovation en usage sont particulièrement fécondes à étudier. Ces objets nouveaux sont en effet susceptibles de donner lieu à des formes de comportements spécifiques. Ils peuvent devenir des "objets-supports » de revendication, des objets intermédiaires (LeviStrauss 1962), traduire à travers des modalités d'appropriation particulières les maux vécus par des collectifs de travail qui ne leur reconnaissent pas un minimum d'efficacité et nous faire entrer dans l'univers des représentations sociales afférentes à ces actions (Lemonnier 1996).

Dans notre exemple, on est frappé par l'usage qui est fait des objets et du contexte immédiat de l'action ${ }^{7}$. Avant d'aller plus loin, il est bon de souligner que les formes de comportement des compagnons, contrairement à ce que pensait la direction, étaient moins liées aux conditions de travail difficiles qu'au rapport aux objets techniques et à leur "affordance " (Gibson 1979), c'est-à-dire à ce qu'ils offrent et procurent aux opérateurs. On ne peut dès lors comprendre les "pins » et les "pin-up ", sans faire référence au TGV, aux amortisseurs et aux images qu'ils renvoient. Les compagnons ne se sentent pas solidaires du record et de cette innovation. Leur travail ne se voit pas: "Quoi de plus banal qu'un amortisseur! Nous n'avons pas l'impression de participer à l'aventure ", disaient-ils fréquemment.

18 L'objet est discret. Contrairement à d'autres corps de métiers qui le remplacent par du neuf, celui-là est réparé, souvent à perte. Ce n'est pas un médiateur entre les hommes et l'innovation. Il n'est pas l'image du progrès que symbolisent le train, sa silhouette et ses performances. Son efficacité technique entre en tension avec son efficacité sociale. 
Les amortisseurs sont de médiocres porte-paroles. Ils ne permettent aucun détournement. Le collectif procède alors à un déplacement dans le choix des signifiants. Il utilise les ressources du contexte immédiat de son activité pour manifester en silence les problèmes qu'il rencontre. Les opérateurs s'appuient sur le potentiel de la situation.

19 Affiches et pins du record sont d'un usage -ou dans ce cas, d'un non-usage- plus facile que les amortisseurs. Ils vont permettre de traduire le malaise. Ils sont à dessein rejetés de l'enceinte de l'atelier de l'établissement, de la vie hors travail, où comme ailleurs, on s'attend à les retrouver. Ils nous permettent d'entrer dans ce que Christophe Dejours (1998 : 113) nomme la " rationalité pathique » : ce qui, dans une action, une conduite ou une décision, relève de la rationalité par rapport à la préservation de soi (santé physique et mentale) ou à l'accomplissement de soi (construction subjective de l'identité). C'est là aussi une façon d'éviter l'aliénation sociale (Sigaut 1990). Cette notion de «rationalité pathique " est l'un des éléments constitutifs de ce que Vinck (1999) nomme la « performance ». Par performance, on entend ce qui est effectivement produit quel qu'en soit le registre: productivité technico-économique, manifestation technique des possibilités insoupçonnées de la machine ou du produit, virtuosité de l'opérateur..., beauté du mouvement machinique, renversement d'un rapport de pouvoir, identité d'un groupe professionnel, exaltante puissance destructrice ou déchaînement de violence dont la technique est capable. (Vinck 1999: 216). Il s'agit bien là d'être efficace pour soi et pour le collectif qu'après Wenger (1998) nous nommerons ici "communauté de pratiques». Selon ce cadre d'analyse et d'interprétation du fonctionnement du collectif, ce sont les pratiques, et notamment les pratiques de nature technique, qui vont fonder la cohérence de la communauté. De ce point de vue, le statut des connaissances dans les communautés de pratique diffère fondamentalement de la conception classique qui prévaut encore en sciences cognitives: la connaissance n'est plus seulement vue comme un ensemble d'informations stockées de manière explicite sous forme de représentations internes ou externes, mais également, et surtout, comme le résultat de la participation active d'acteurs à un processus social de construction du sens. Cette manière de considérer le statut des connaissances, si l'on y adhère, invalide donc automatiquement une démarche qui se donnerait par exemple pour objectif d'identifier les connaissances techniques mises en œuvre dans un collectif, indépendamment des conditions sociales de leur production (Geslin \& Salembier 2002).

Les calendriers, les pins et le jeu d'échecs sont autant d'objets et de modes d'expression qui, aux yeux des observateurs que nous étions, traduisaient le malaise ambiant. Ils étaient porteurs de sens, mais les comportements auxquels ils donnaient lieu n'avaient pas attiré l'attention de l'encadrement qui s'était investi dans la mise en œuvre de modifications techniques, remplaçant ici une machine, transformant là un poste de travail. Ces postes nécessitaient bien sûr des aménagements ${ }^{8}$, il fallait aussi améliorer les conditions de travail des opérateurs dont l'activité générait, entre autres, de nombreux accidents. Le nombre d'accidents aux mains, par exemple, était de $40 \%$ supérieur au nombre total d'accidents dans les autres ateliers, les tendinites du poignet étaient fréquentes, dues à des gestes répétitifs. L'absentéisme allait croissant. De 330 jours en 1989, il était passé à 460 en 1990. Les aménagements de l'atelier envisagés et réalisés par l'encadrement se concentraient sur les aspects matériels, évacuant de fait les dimensions cognitives de l'activité. Le problème se situait au-delà de l'atelier, dans le rapport à l'innovation. La place occupée par le fruit du travail (les amortisseurs), et 
ses représentations inhérentes, n'étaient pas suffisamment efficaces pour que le collectif se retrouve dans cette aventure technologique.

21 La description du travail fournie par l'encadrement était de type "gestionnaire" (Dejours 1998: 64). Ce type de description est donné par les services des méthodes, par le service de la qualité et par le service de gestion des ressources humaines. Si l'on se réfère à notre exemple, la description gestionnaire souligne que le processus est efficace dans la mesure où les objectifs fixés, en dépit du coût physique et cognitif, sont atteints par les compagnons de l'atelier. Les amortisseurs ressortent à l'autre bout de la chaîne, réparés, prêts au remontage. Cette description est détachée des modalités réelles $^{9}$ de travail au sein du collectif. Elle se distingue de la " description subjective » du travail (Déjours 1998), qui est reconstruite à partir du récit des opérateurs (récit des difficultés des uns et des autres dans l'exercice de leur travail; récit aussi des façons de "s'arranger" avec ces difficultés, de les surmonter ou de les contourner). Elle nous renvoie aux problèmes rencontrés par ce collectif pour remplir ses objectifs et se positionner par rapport à l'innovation lorsqu'elle n'est pas encore une simple technique en usage. Cette description subjective ne livre pas tout. À aucun moment de nos enquêtes, les compagnons n'avaient fait référence aux usages implicites auxquels donnaient lieu les affiches et les pins. Nous avons dû remonter la chaîne, comparer les situations entre divers ateliers, faire émerger le sens de ces "non-présences » dans le contexte immédiat et quotidien. Les formes d'activités que sont ces revendications prennent place en marge de l'activité technique. Elles trouvent toutefois leur source dans cette activité et sont proches de ce que les ergonomes nomment «l'activité métafonctionnelle ", et qui n'est pas directement orientée vers la production immédiate. Elle a trait à la construction de connaissances ou d'outils (matériels ou cognitifs) destinés à une éventuelle utilisation ultérieure, et pour notre propos, vise à faciliter l'exécution de la tâche ou à améliorer la performance : en quelque sorte à être plus efficace pour soi et/ou le milieu qui nous entoure. Mais pour que cette notion soit opérationnelle, il est nécessaire de lui intégrer des dimensions politiques et de « revendication discrète » qu'elle néglige.

La notion de jugement intervient aussi en arrière-plan dans la construction sociale de «l'efficacité ». Dire qu'un acte est efficace, c'est porter un jugement sur autrui ou sur soi-même. Travailler dans cet atelier est associé à une forme de punition par les membres de l'établissement, mais aussi par les compagnons eux-mêmes. Ils ont longtemps souffert de l'absence de reconnaissance par leur hiérarchie, mettant en évidence un manque de communication tant au niveau des simples relations humaines qu'à celui de l'écoute et de la prise en compte de leurs problèmes. Le jugement d'autrui a des répercussions directes sur l'activité du collectif de l'atelier d'amortisseurs. Sans faire de cet événement plus que ce qu'il représente, on peut considérer le tournoi d'échecs qui a lieu après chaque repas, comme un facteur de cohésion du collectif, mais aussi comme un des moyens permettant de sortir de l'image de " mauvais élèves » qui leur est associée et par conséquent de modifier le jugement d'autrui. De même, la nonappropriation des objets qui traduisent en général l'adhésion à l'innovation est aussi une façon de soumettre ces revendications discrètes au jugement des collègues de l'établissement, revendications dont on espère qu'elles permettront d'atteindre le but que l'on s'est fixé (efficacité pour soi), d'être suffisamment efficace pour attirer l'attention de l'encadrement sur la situation.

Les objets sont notre plomb dans la tête 
Aborder la délicate notion d'efficacité, en ethnologie des techniques, c'est accepter de sortir des cadres d'analyse qu'elle nous livre et prendre en compte des séries de concepts produits ailleurs, pour saisir un peu plus de son essence. Le malentendu sur lequel reposent les échanges entre Lemonnier et Latour, comme de fait entre une forme de technologie culturelle et la nouvelle sociologie des sciences et des techniques, tient aussi à l'absence d'une dissociation claire entre technique et innovation. La différence est grande en effet entre l'étude des techniques d'un usage largement répandu et celle qui traite de l'émergence de techniques nouvelles. Dans ce dernier cas, il faut aussi, comme nous l'avons fait dans cet article, distinguer la phase d'émergence de l'innovation de celle de son usage en tant que tel, qui ne dure qu'un temps avant son objectivation (Descola 1994) et sa stabilisation dans la société. Une objectivation qui l'amène progressivement au statut de simple technique. Les formes d'efficacités en actions sont étroitement dépendantes de la phase d'appropriation dans laquelle se trouve l'objet considéré. Les compagnons de l'atelier, n'auraient sans doute pas pu agir de la même façon s'il n'y avait pas eu ce record de vitesse. Ils n'auraient pas pu déléguer aux pins et aux « pin-up » les formes de malaise qu'ils ressentaient. Le record arrivait à point nommé. Les formes d'efficacités qui en découlent sont spécifiques de ce moment particulier où les habitudes et les routines liées à la technique refont sa place à ce que Tisseron (1999) nomme "l'émerveillement qui accompagne notre découverte de chaque nouvel objet ». L'émerveillement, dans ce cas, s'efface au profit d'autres formes sociales d'appropriations de l'objet technique. Il ouvre la porte des possibles, donnant aussi naissance aux revendications des cheminots, aux interrogations de leur encadrement.

Parler d'efficacité, c'est aussi parler des hommes, des actes qui en rendent compte, et des usages qui sont faits de leur contexte immédiat. Une action peut être jugée efficace si on la sort de son contexte. Les compagnons réparent en effet les amortisseurs et remplissent les objectifs qu'ils se sont fixés et qui sont fixés par leur encadrement. Dès que cette activité qualifiée d'efficace est située dans son contexte et, de fait, associée aux éléments de ce contexte, le jugement qui est porté prend une autre dimension. C'est moins alors la seule activité de réparation des amortisseurs qu'il faut prendre en compte, que les traces qui nous sont livrées par l'étude de son contexte immédiat dans sa relation avec un contexte élargi peuplé d'humains et d'objets divers comme les affiches du record et les pins. Ce sont bien les comportements implicites, dans leur relation aux objets en présence, qu'il s'agit de faire émerger à partir d'un corpus de concepts issus de disciplines plus enclines à nous révéler un peu ce que cette notion recouvre, faisant d'une évidence un objet de recherche dont la complexité est encore à explorer.

\section{BIBLIOGRAPHIE}

Akrich, Madeleine

1994 «Comment sortir de la dichotomie technique/société. Présentation des diverses sociologies 
de la technique ", pp. 105-131, in B. Latour et P. Lemonnier (eds), De la préhistoire aux missiles balistiques. L'intelligence sociale des techniques. Paris : La Découverte.

Cohen, Yves \& Dominique Pestre

1998 « Présentation », Annales 4-5 : 721-744.

Dejours, Christophe

1998 Souffrance en France. Paris : Point/Seuil.

Descola, Philippe

1994 « Pourquoi les Indiens d'Amazonie n'ont-ils pas domestiqué le pécari ? », pp. 329-344, in B. Latour et P. Lemonnier (eds), De la préhistoire aux missiles balistiques. L'intelligence sociale des techniques. Paris : La Découverte.

Edgerton, David

1998 « De l'innovation aux usages. Dix thèses éclectiques sur l'histoire des techniques », Annales $4-5: 815-837$.

Geslin, Philippe

1998 Compte rendu de l'ouvrage de R. Cresswell, Prométhée ou Pandore ? Propos de technologie culturelle. Paris, Éditions Kimé, 1996, in L'Homme 147 : 262-264.

1999 L'Apprentissage des mondes. Une anthropologie appliquée aux transferts de technologies. Paris :

Éditions de la Maison des sciences de l'homme.

Geslin, Philippe \& Olivier Berzanne

1991 Étude ergonomique du chantier SNCF de réparation des amortisseurs de l'atelier de B. Mémoire d'ergonomie. Paris : CNAM.

Geslin, Philippe \& Pascal Salembier 2002 « La fleur, l'épice. Usage social du corps et communauté de pratique dans la relance de produits authentiques ", Anthropologie et connaissances.

Gibson, James J.

1979 The Ecological Approach to Visual Perception. Boston : Houghton Mifflin Company.

Jullien, François

1996 Traité de l'efficacité. Paris : Grasset (« Le Livre de Poche, Biblio essais »).

Latour, Bruno

1996 « Lettre à mon ami Pierre sur l'anthropologie symétrique », Ethnologie Française XXVI (1) : $32-37$.

Latour, Bruno \& Pierre Lemonnier (eds)

1994 De la préhistoire aux missiles balistiques. L'intelligence sociale des techniques. Paris : La

Découverte.

Lemonnier, Pierre

1996 « L'ethnologie des techniques et les objets industriels », Ethnologie Française XXVI (1) : 17-31.

Lévi-Strauss, Claude.

1985 La Pensée sauvage. Paris : Presse Pocket (1ère édition 1962).

Pavard, Bernard \& Laurent Karsenty

1997 « Différents niveaux d'analyse du contexte dans l'étude ergonomique du travail collectif », Réseaux 85 : 73-100.

Pieyre de Mandiargues, André

1956 « Le feu, la pierre », in Hommage à Françis Ponge. Paris : Gallimard (« NRF »). 
Ponge, Francis

2000 Le Parti pris des choses. Paris : Gallimard (1ère édition 1942).

Quéré, Louis

1997 « La situation toujours négligée », Réseaux 85 :163-192.

Segalen, Martine \& Christian Bromberger

1996 « L'objet moderne : de la production sérielle à la diversité des usages ", Ethnologie Française

XXVI (1) : 5-16.

Sigaut, François

1990 « Folie, réel et technologie », Techniques \& culture 15 : 167-179.

Suchman, Lucy-A.

1987 Plans and Situated Actions : The Problem of Human-Machine Interaction. Cambridge : Cambridge University Press.

Tisseron, Serge

1999 Comment l'esprit vient aux objets. Paris : Aubier.

Vinck, Dominique

1999 Ingénieurs au quotidien. Grenoble : Presses Universitaires de Grenoble.

Wenger, Etienne

1998 Communities of Practice - Learning, Meaning and Identity. Cambridge MA : Cambridge university Press.

\section{NOTES}

1. Les travaux de Pierre Lemonnier s'inscrivent dans une démarche qui ne dissocie pas véritablement l'ethnologie des techniques de l'ethnologie au sens strict, alors que toute la démarche de R. Cresswell par exemple tend selon nous à faire de la technologie culturelle un champ disciplinaire spécifique au sein de l'ethnologie.

2. B. Latour souligne dans sa « Lettre à mon ami Pierre » : «C'est bien pourquoi depuis vingt ans [...] nous avons décidé de refaire les sciences sociales de fond en comble pour qu'elles encaissent, absorbent, digèrent les non-humains, c'est-à-dire justement les objets, les biens, les matières, les universaux, les contraintes que la sociologie classique avait laissés hors de son champ depuis Comte ou réuni, sous une forme hautement critiquable, en une infrastructure matérielle. » (1996:33).

3. Ébauche que nous avions affinée dans L'apprentissage des mondes... (Geslin 1999).

4. Après Jullien (1996: 48), nous reprenons la notion de plan d'action : « Tout projet élaboré comportant une suite ordonnée d'opérations, en tant que moyens, destinée à atteindre le but visé ».

5. Jullien (1948: 50) entend par " application », la théorie conçue d'avance venant recouvrir le réel, de façon à pouvoir ensuite le calquer sur elle.

6. Serge Tisseron (1999: 10) écrit cette phrase que nous reprenons à notre compte : «L'émerveillement qui accompagne chaque nouvel objet cède rapidement la place aux habitudes ».

7. L'ensemble des ressources contingentes à l'activité des opérateurs, exploitées pour donner sens à la situation de travail et y répondre de la façon la plus adéquate possible (Pavard \& Karsenty 1997 : 75). Le contexte est le champ en fonction duquel une action, un geste, une parole, un événement ou un objet acquièrent une intelligibilité, un sens, 
une individualité. C'est aussi l'ensemble des éléments singuliers (d'information, de savoir, de sens, de perception) sur lesquels on se règle pour produire une action et revendiquer son caractère approprié (Quéré 1997 : 184).

8. Pour une analyse détaillée de l'activité et un diagnostic ergonomique, voir Geslin \& Berzanne (1991).

9. Le réel est défini comme ce qui résiste aux connaissances, aux savoirs, aux savoirfaire et d'une façon plus générale à la maîtrise. L'expérience du réel dans le travail se traduit par la confrontation à l'échec (Dejours 1998 : 81).

\section{RÉSUMÉS}

La notion d'efficacité sous-tend l'ensemble des travaux d'ethnologues ou de sociologues qui traitent de la construction conjointe des faits sociaux et des faits techniques. En prenant comme base de réflexion les échanges qui eurent lieu en 1996 autour de cette notion, entre l'ethnologue Pierre Lemonnier et le sociologue Bruno Latour, cette contribution se propose d'étendre le débat. À travers l'ethnographie d'un atelier de réparation des amortisseurs du Train à Grande Vitesse (TGV), nous sommes amené à sortir des cadres théoriques qui nous sont proposés par l'ethnologie et la sociologie des techniques. La perception occidentale classique de "l'efficacité » en termes de rapport de moyens à fin trouve ici ses limites. Elle projette un plan sur le cours des choses. Les conduites résultent d'une application quand en fait dans notre exemple, on est conduit à les considérer en termes d'exploitation, c'est-à-dire en tant qu'utilisation du potentiel impliqué dans la situation.

Objects knock some sense into us. Efficiency in acts and using innovations.

The notion of efficiency underlies sociological and anthropological studies which deal with the conjoined construction of social and material facts. On the basis of discussions that an anthropologist, Pierre Lemonnier, and a sociologist, Bruno Latour, exchanged in 1996 about this notion, this article widens the debate. Through a case study of a shock-absorber repair shop of the "very high speed train» (TGV), we are led to go beyond the theoretical scopes of anthropology and sociology of techniques. The classical western sense of efficiency as a relation between means and purposes meets its limits. It projects a planning on the course of things. Behaviours result from an application whereas we consider them as an exploitation, i.e., as a potential in the situation.

Los objetos nos hacen poner los pies en la tierra. Eficacia en las acciones, innovaciones en los usos.

Sobre la noción de eficacia se apoyan el conjunto de trabajos de etnólogos y sociólogos que tratan acerca de la construcción conjunta de los hechos sociales y de los hachos técnicos. Tomando como base para la reflexión el intercambio de ideas que en torno a esta noción tuvo lugar en 1996 entre el etnólogo Pierre Lemonnier y el sociólogo Bruno Latour, este texto se propone ampliar el debate. A través de la etnografía de un taller de reparación de amortiguadores del Tren de Alta Velocidad (TGV), nos vemos empujados a salir de los cuadros teóricos que nos proponen la etnología y la sociología de las técnicas. La percepción occidental clásica de "eficacia ", en términos de la adecuación entre medios y fines, encuentra aquí sus límites. Esta percepción proyecta un plan sobre el devenir de las cosas. Se supone así que las conductas resultan de una 
aplicación cuando de hecho en nuestro ejemplo, nos vemos llevados a considerar dichas conductas como explotación, como utilización de un potencial que ya está implicado en una situación dada.

INDEX

Mots-clés : cognition distribuée, communauté de pratique, Latour (B.), Lemonnier (P.), objets intermédiaires, rationalité pathique

Keywords : Community of practices, intermediary objects, pathic rationality, shared cognition, TGV

\section{AUTEUR}

\section{PHILIPPE GESLIN}

INRA - SAD, Chemin de Borde Rouge, Auzeville, BP 2731326 Castenet Tolosan Cedex. 